\title{
INITIAL CLINICAL STAGING AND INCIDENCE OF MOLECULAR SUBTYPE IN BREAST CANCER PATIETS TREATED FROM JAN/2011 TO DEC/2019 AT PÉROLA BYINGTON HOSPITAL
}

Mauro Orlando Meurer Oliveira', Jorge Yoshinori Shida', André Mattar ${ }^{1}$, Felipe Andreotta Cavagna', Luiz Henrique Gebrim

${ }^{1}$ Centro de Referência da Saúde da Mulher - São Paulo (SP), Brazil.

Introduction: Clinical staging (CS) has great importance for therapeutic programming and prognostic evaluation in patients diagnosed with breast cancer. Malignant breast tumors can be classified according to their immunohistochemical (IHC) profile. The study of the IHC profile can also assist in public health strategies, since they determine therapeutic planning. Objectives: Compare our database with literature data. Methods: The staging database (TNM, Tumor, Nodes and Metastasis) of the CRSM-SP (Pérola Byington Hospital) of patients with breast cancer treated at this institution from January 2010 to December 2019. Results: It was observed that $5.7 \%$ of the patients had in situ tumors. In the invasive form of tumors, $22.7 \%$ of the patients were diagnosed in CS I. Stage II was the one with the highest occurrence, corresponding to $36.5 \%$. Advanced cases belonging to stages III and IV respectively represented $28.2 \%$ and $3.26 \%$. As for the IHC profile of our 10,665 patients, luminal tumors A represented 24.7\%, luminal B 32.7\%. Patients with overexpressed HER2 were subdivided into pure HER2 (7.7\%) and triple positive (9.8\%). Triple negative tumors represented $25.1 \%$ of patients. A Brazilian study with SUS (Brazilian Unified Health System) data included 201,079 women: 19.5\% were in stage I, 40.4\% in stage II, 30.9\% in stage III, and 9.3\% in stage IV. In comparison with the public health system in England, a population study of 86,852 cases of breast cancer found $37 \%$ of diagnoses in stage I, 32\% in CS II, $8 \%$ in CS III, and 5\% in CS IV. The high ratio of patients (17\%) in an unknown staging is noteworthy. In the USA, in a population study of 320,124 women, $72.6 \%$ were classified as luminal A, $11.2 \%$ as luminal B, $4.8 \%$ with overexpression of HER2 and 11.3\% as triple-negative. Data from a Brazilian publication with 2,461 patients observed luminal A in $28.8 \%$ of the cases, luminal B 39.5\%, pure HER2 7.9\%, triple positive $9.7 \%$ and triple-negative were $14 \%$, with the exception of triple-negative, similar to our results. It is possible that the higher ratio of patients with triple negative tumors at CRSM is explained by the higher incidence of non-screened patients, aged under 50, which are about $39 \%$. Conclusions: The finding of $28.4 \%$ of in situ tumors in stage I shows the results of an occasional screening. The predominance of patients in stage II (40.4\%) shows the predominance of palpable tumors in our population and the importance of making an agile diagnosis, preventing progression to stage III. The predominance of luminal tumors $68.3 \%$ and HER $2(16.7 \%)$ were similar to those in the literature. However, the ratio of $25.1 \%$ of triple-negative patients contributes to higher mortality in Brazil and requires greater diagnostic and therapeutic agility. 